\title{
Improving the cardiometabolic health of people with psychosis: A protocol for a randomised controlled trial of the Physical Health Nurse Consultant service
}

\author{
Brenda Happell $^{\mathrm{a}, *}$, Jackie Curtis ${ }^{\mathrm{b}}$, Michelle Banfield ${ }^{\mathrm{c}}$, John Goss ${ }^{\mathrm{d}}$, Theophile Niyonsenga ${ }^{\mathrm{d}}$, \\ Andrew Watkins ${ }^{\mathrm{e}}$, Chris Platania-Phung ${ }^{\mathrm{a}}$, Lynelle Moon ${ }^{\mathrm{f}}$, Philip Batterham ${ }^{g}$, Brett Scholz ${ }^{\mathrm{h}}$, \\ Vanessa Prescott ${ }^{\mathrm{i}}$, Robert Stanton ${ }^{\mathrm{j}}$ \\ a School of Nursing and Midwifery, University of Newcastle University Drive Callaghan New South Wales, 2308, Australia \\ b Youth Mental Health, South Eastern Sydney Local Health District and Conjoint Senior Lecturer, University of New South Wales, Australia \\ ${ }^{\mathrm{c}} \mathrm{Head}$, ACACIA: The ACT Consumer \& Carer Mental Health Research Unit, Centre for Mental Health Australian National University, Australia \\ ${ }^{\mathrm{d}}$ UC Health Research Institute, University of Canberra, Australia \\ e Co-clinical lead - Keeping the Body in Mind Program, NSW Health, Australia \\ ${ }^{\mathrm{f}}$ Head, Health Group, Australian Institute for Health and Welfare, Australia \\ ${ }^{g}$ Centre for Mental Health Research, Research School of Population Health, Australian National University, Australia \\ ${ }^{\mathbf{h}}$ ANU Medical School, College of Health and Medicine, The Australian National University, Australia \\ ${ }^{i}$ Senior Data analyst/project manager, Australian Institute of Health and Welfare, Australia \\ ${ }^{\mathrm{j}}$ Research Cluster Leader - Muscular and Skeletal Health, School of Health, Medical and Applied Sciences, Central Queensland University, Australia
}

\section{A B S T R A C T}

Background: Over 690,000 Australians experience psychosis annually, sig-

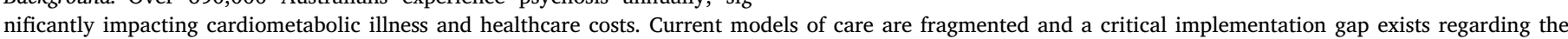
delivery of coordinated physical healthcare for Australians with psychosis.

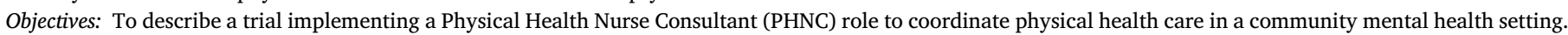

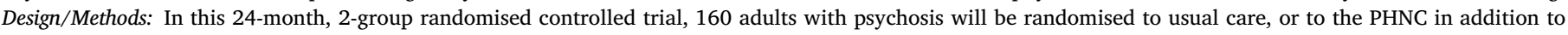

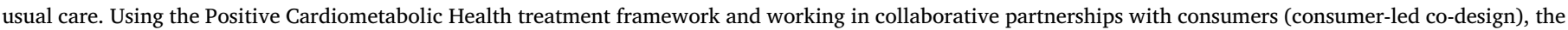

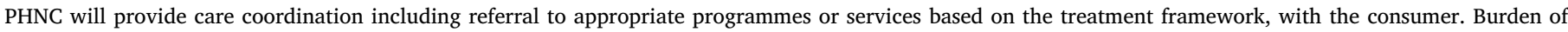

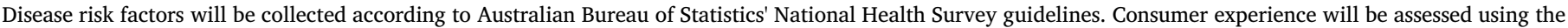

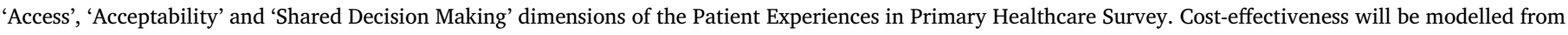
Burden of Disease data using the Assessing Cost Effectiveness Prevention methodology.

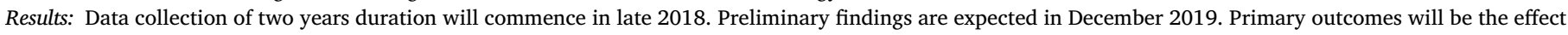
of the PHNC role on physical healthcare in community-based adults with psychosis.

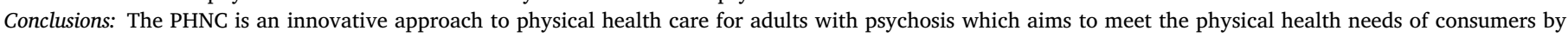
addressing barriers to physical health care.

\section{Introduction}

Inadequate cardiometabolic care of mental health consumers contributes to 12,000 deaths a year in Australia [1]. Consumers with psychosis have a 2-3 fold higher risk of developing physical health disorders compared to the general population $[2,3]$, and experience a mortality gap of between 10 and 25 years [3,4]. Physical comorbidities are responsible for $80 \%$ of excess deaths, at a cost of $\$ 15$ billion annually $[5,6]$. In light of the Department of Health's Fifth National Mental Health Plan priorities including improving the physical health of people living with mental illness and reducing early mortality, these data provide strong ethical and economic rationale for improving

\footnotetext{
* Corresponding author.

E-mail addresses: Brenda.happell@newcastle.com (B. Happell), Jackie.Curtis@health.nsw.gov.au (J. Curtis), Michelle.banfield@anu.edu.au (M. Banfield), John.Goss@canberra.edu.au (J. Goss), John.Goss@canberra.edu.au (T. Niyonsenga), Andrew.Watkins@health.nsw.gov.au (A. Watkins), Lynnelle.Moon@aihw.gov.au (L. Moon), Philip.Batterham@anu.edu.au (P. Batterham), brett.scholz@anu.edu.au (B. Scholz), Vanessa.prescott@aihw.gov.au (V. Prescott), r.stanton@cqu.edu.au (R. Stanton).
} 
physical healthcare in this population $[7,8]$.

Community mental health services represent an important setting for coordinating physical healthcare $[9,10]$, with clinical guidelines, including those in an Australian context, recommending consumers receive regular cardiometabolic health monitoring [11-13]. Cardiometabolic monitoring alone is not sufficient with rates of routine assessment between 7 and $63 \%$, and between 6 and $60 \%$ of consumers referred for additional health services [14,15]. Limited referral and access to appropriate healthcare services $[6,16]$, and a lack of care coordination and follow-up $[1,16]$ compounds this problem.

There are multiple barriers to improving consumer cardiometabolic health $[1,16]$. Mental healthcare professionals cite limited time for follow-up and lack of knowledge of referral pathways as limiting cardiometabolic monitoring and subsequent action [14]. Inadequate specific expertise in cardiometabolic health results in priority given to the treatment of symptoms and risks associated with mental illness [17]. Consumers frequently experience discrimination, stigma and low treatment expectations by healthcare providers, which inhibits help seeking [17]. Coupled with a fragmented health system and a lack of continuity of care, this means many consumers do not actively seek cardiometabolic care [18]. This significant service implementation gap has prompted clinical, consumer and government groups to call for new models of proactive integrated cardiometabolic monitoring, management and care coordination to address barriers [1,8].

One strategy with the potential to address the systemic, clinical and individual barriers that lead to comorbid physical illness and early mortality is the implementation of a Physical Health Nurse Consultant (PHNC) service. In the Australian context, nurse-led models of physical health care have been demonstrated as effective at increasing the rates of cardiometabolic monitoring [15,19] but evidence is limited. The PHNC is a dedicated mental health nurse with specialist skills in cardiometabolic health and co-located within mental health services to clarify lines of responsibility, creating a single point of contact for relationship building with consumers. Consistent with consumer co-design and person-centred care, the PHNC could embed shared decisionmaking, and improve service navigation and coordinated utilisation of existing health and medical resources [20]. In this paper, we describe the protocol for the implementation and evaluation of a PHNC service to be delivered alongside usual care for consumers with psychosis. In particular, we will evaluate the PHNC service relative to usual care with respect to cardiometabolic health, consumer experience of care, and cost-effectiveness.

\section{Methods}

\subsection{Design}

1. This randomised controlled trial will examine the impact of a PHNC service in a community-based mental health service in a large Australian metropolitan city. Specifically, this project will evaluate whether the PHNC service (relative to usual care alone) results in improvements in:

2. Cardiometabolic health based on Burden of Disease risk factors: Body Mass Index (BMI), total cholesterol, fasting glucose, systolic blood pressure, smoking, second hand smoke, fruit intake, vegetable intake, daily alcohol intake, binge drinking, physical inactivity;

3. Consumer experience of care: experience, acceptability, shared decision-making, quality of life;

4. Cost-effectiveness of physical health service delivery.

We hypothesise that relative to usual care, participants randomised to the PHNC service will experience;

- a greater reduction in burden of disease risk factors,

- greater access to and acceptability of physical healthcare, shared decision-making, and quality of life.
Additionally we hypothesise the PHNC intervention will be costeffective. The project will take approximately 36-months. The intervention will be a prospective single site 24 -month, 2 -arm randomised controlled trial. The 24-month time point provides assessment of any long-term changes in health status resulting from the intervention. Burden of Disease modelling and cost-effectiveness analysis will follow the intervention in month 31 and take approximately 2 months to complete. The intervention is grounded in the Integrated Theory of Health Behaviour Change [21] which draws on theories of health behaviour change, self-regulation and social support, and findings from studies of self-management of chronic illness. The study will employ a Clinical Research Assistant, and a Physical Health Nurse Consultant for specific roles in the study, as described below.

Ethical clearance for this study has been received from the Australian Capital Territory (ACT) Health (Application ID: 2018/ ETHOOO29) and the study is registered with the Australian and New Zealand Clinical Trials Registry (ACTRN12618000678291p).

\subsection{Eligibility and recruitment}

All consumers aged 18-65, diagnosed with a DSM-5 psychotic disorder who attend a large community mental health service in an Australian capital city will be eligible to participate. The service has 14 case managers and 385 eligible consumers. Consumers will be excluded if they are unable to speak and read English, or unable or unwilling to provide informed consent. After providing informed consent and completing the baseline assessment, participants will be randomly allocated to receive the PHCN intervention or Usual Care control, with the randomisation process and interventions described below.

Based on clinic caseload, approximately 13 participants per arm are expected to enter the intervention each week over 6-weeks' rolling recruitment. This recruitment rate is feasible as an average of 64 unique consumers attend the service each week over a $2-3$ month contact cycle. Overall, recruiting 160 participants is feasible as it represents $41 \%(160 / 385)$ of eligible consumers that typically attend the service over such a period. This is the most feasible and realistic method of participant recruitment as potential participants will already be attending the service for scheduled appointments.

\subsection{Randomisation and blinding}

After completing baseline assessments, participants will be randomised to the intervention or control group by a team member not involved in screening (eligibility and entry of participants) or data collection (baseline and follow-up assessments) using the process of permuted block randomisation (stratified on age and gender using computer-based allocation), to ensure balance between groups (intervention and control groups). Allocation sequence will be use a webbased automated computer-generated randomisation system. Randomisation will be stratified by gender and age group, with block sizes of either 4, 6 or 8, and conducted in accordance with international guidelines [22]. Allocation concealment will be achieved using sequentially numbered opaque sealed envelopes. Investigators and clinicians assessing outcomes will be ignorant of future treatment allocation and will have no control over the order of patients randomised into the trial. Participants and data collectors (outcomes assessors) will be blinded to the treatment allocation until after baseline assessments are completed. Team members conducting data analysis will not be involved in data collection, and treatment allocations will be blinded until after data are analysed.

Usual Care group participants will receive usual care from their case manager. Based on previous research, it is anticipated only around 3\% of this group will receive cardiometabolic monitoring while receiving usual care [15]. Participants randomised to the PHNC group (intervention) will attend an initial consultation with the PHNC lasting approximately $45 \mathrm{~min}$ that includes interpretation of cardiometabolic 
Table 1

Australian Institute for Health and Welfare Burden of Disease risk factors.

\begin{tabular}{|c|c|c|}
\hline Risk Factor & Measurement method & Base risk level \\
\hline Elevated BMI & Measurement of height and weight & $21-23 \mathrm{~kg} \cdot \mathrm{m}^{-2}$ \\
\hline Total cholesterol & Blood test $5.5 \mathrm{ml}$ & Mean 3.8-4.0 mmol. $\mathrm{L}^{-1}$ \\
\hline Fasting glucose & Blood test, $5.5 \mathrm{ml}$ & Mean 4.9-5.3 mmol.. ${ }^{-1}$ \\
\hline Systolic blood pressure & Measurement & Mean $110-115 \mathrm{mmHg}$ \\
\hline Smoking & Self-reported current smoking & No exposure to tobacco smoking \\
\hline Second hand smoke & Self-reported exposure to smoke in the home at least daily & No exposure to second hand smoke \\
\hline Fruit intake & Self-reported serves of fruit per day & 2 servings per day \\
\hline Vegetable intake & Self-reported serves of vegetables per day & 5 servings per day \\
\hline Alcohol use: daily intake & Self-reported average consumption of alcohol per day & No alcohol consumption \\
\hline Alcohol use: binge drinking & Self-reported consumption of $0.06+\mathrm{kg}$ of alcohol on a single occasion & $<0.06 \mathrm{~kg}$ alcohol on a single occasion \\
\hline Physical inactivity & Self-reported metabolic equivalent of tasks (METs) & $\leq 8000$ METs.week $^{-1}$ \\
\hline
\end{tabular}

assessment data, and risk management and care coordination as guided by the Positive Cardiometabolic Health treatment framework [23]. After the initial meeting, participants will attend quarterly consultations and shorter $(\sim 10 \mathrm{~min})$ monthly telephone follow-ups for the remainder of the intervention to track progress against agreed goals. The PHNC intervention will be delivered alongside usual care for their mental health condition.

\subsection{Role of the Clinical Research Assistant}

The Clinical Research Assistant (CRA) will be a registered nurse employed for a period of two and a half years on a full-time basis. The CRA will collect assessment data including clinical and self-report measures for participants in both arms of the study. The CRA will not discuss the intervention with participants randomised to the usual care group and this will reduce contamination effects. Risk Factor (BMI, total cholesterol, fasting glucose, systolic blood pressure, smoking, second hand smoke, fruit intake, vegetable intake, daily alcohol intake, binge drinking, physical inactivity) and Consumer Experience (access, acceptability, shared decision making, quality of life) data will be collected from both groups by the CRA at baseline and repeated at interim points in months $3,6,9,12,15,18$ and 21 with a final assessment in month 24. This frequency is critical for the intervention as the PHNC uses these data to calculate changes in risk factors and make appropriate preventative shared decisions with PHNC group participants based on the Positive Cardiometabolic Health treatment framework [23]. This timing is also consistent with guidelines for chronic conditions such as diabetes [24].

\subsection{Role of the PHNC}

The PHNC will be a mental health nurse with specialised training in physical health care and employed for a period of two and a half years on a full-time basis. Usual Care group participants will receive usual care from their case manager. Based on previous research, it is anticipated only around $3 \%$ of this group will receive cardiometabolic monitoring while receiving usual care [15]. Participants randomised to the PHNC group (intervention) will attend an initial consultation with the PHNC lasting approximately $45 \mathrm{~min}$. After the initial meeting, participants will attend quarterly consultations and shorter $(\sim 10 \mathrm{~min})$ monthly telephone follow-ups for the remainder of the intervention to track progress against agreed goals. The PHNC intervention will be delivered alongside usual care for their mental health condition.

The PHNC will perform the primary roles of the intervention as described below.

- Risk management: During a consultation with the consumer, the PHNC will use the Positive Cardiometabolic Health treatment framework [23] to the assessment data collected by the CRA, and any additional information discussed with the consumer. This framework considers the clinical and behavioural risks identified as part of the cardiometabolic assessment, along with the consumer's selfidentified needs, goals and health priorities, shared decision making with the consumer, and local-availability and consumer-acceptability of management options;

- Care coordination: Once the PHNC and consumer agree on an approach to physical healthcare, the PHNC provides care coordination including supported referral to appropriate programmes or services (GPs, dietitians, nutritionists, accredited exercise physiologists, smoking cessation programs and other available services) as recommended by the treatment framework, communicates relevant clinical history, provides appointment reminders, and timely followup of results.

\subsection{Measures}

\subsubsection{Cardiometabolic health}

Risk factor outcome data for 11 contributing risk factors for the Australian Institute for Health and Welfare (AIHW) Burden of Disease (BoD) analysis (see Table 1) will be collected. [25]. The risk factor data enables the calculation of the reduction in Disability-adjusted life years (DALYs) in the intervention group that will occur in all those diseases which are affected by the 11 risk factors This approach ensures accurate capture of the most significant disease risk factors, using established methodologies that are validated for use in populations with mental illness [26]. These data will also be used in the cost-effectiveness analysis to model changes in service utilisation and cost resulting from the PHNC intervention. Further, these measures allow us to compare outcomes with influential datasets including the AIHW's Burden of Disease Study, the Australian Survey of High Impact Psychosis, and evaluations of the HeAL framework [12].

At each time point, the CRA will collect clinical measures including BMI, total cholesterol, fasting glucose, and blood pressure. Additional health behaviour measures such as smoking, second hand smoke, fruit intake, vegetable intake, alcohol use: daily intake, alcohol use: binge drinking, and physical inactivity will be collected by self-report using 23 relevant questions from the National Health Survey. Examples of relevant questions include:

- How many serves of vegetables do you eat each day?

- Do you currently smoke?

- In the last week, how many times have you walked continuously, for at least $10 \mathrm{~min}$, for fitness, recreation or sport?

This is anticipated to take $\sim 5-10$ min to complete.

\subsubsection{Acceptability}

Consumer experience and acceptability are a central component of health policy that measures the performance of health services' responsiveness to consumer expectations [20]. We will track consumer experience using four outcome measures (access, acceptability, shared decision making, quality of life). Access to appropriate preventative 
services will be measured using the 'Access' $(\alpha=0.92)$ dimension of the Patient Experiences in Primary Healthcare Survey (PEPHCS) [27] which details economic and ongoing access to the intervention and referred services. Acceptability of services will be measured using the 'Patientreported impacts of care' $(\alpha=0.84)$ dimension of the PEPHCS which rates patient activation, safety and confidence in the system. Shared decision-making will be measured using the 'Interpersonal communication' $(\alpha=0.75)$ dimension of the PEPHCS which rates communication, respectfulness and shared decision-making. Together, these three dimensions include 38 items that take $\sim 5-10$ min to complete. Quality of life will be measured using Assessment of Quality of Life (AQoL)-8D [28] utility scores. AQoL-8D is a 35-item instrument that is sensitive for use in mental health populations [29] and has high reliability ( $\alpha=0.96$ ) and good content validity [30].

\subsubsection{Cost-effectiveness}

Cost-effectiveness analysis will be conducted to support the translational and policy impact of the PHNC service. Cost-effectiveness will be determined using the Assessing Cost Effectiveness (ACE) Prevention methodology [31-33] and in accordance with Pharmaceutical Benefits Advisory Committee economic evaluation guidelines [34]. Using AIHW BoD models, between-group differences in Disability Adjusted Life Years (DALYs) over the next 20 years for each disease (based on changes in the 11 risk factors at 24 months) will be calculated to derive an estimate of total DALYs saved due to the intervention. Betweengroup differences in service use and expenditure over the next 20 years will be calculated by assuming expenditure on services is proportional to disease prevalence. Therefore a $5 \%$ reduction in disease prevalence (based on PHNC-attributed risk factor changes) would reduce service use and expenditure by $5 \%$. This will allow long-term changes in service utilisation and cost associated with admitted patient services, out-ofhospital medical services and prescription pharmaceuticals to be estimated. Between-group differences in quality of life $(A Q O L-8 D)$ utility scores at 24 months will be used to estimate the short-term impact of the PHNC intervention on 'burden severity' in people with psychotic disorders. The total cost of the intervention minus the estimated health system cost savings attributed to the intervention (calculated above) will then be used in combination with the estimated reduction in DALYs to calculate the cost-effectiveness ratio (cost per DALY) for the PHNC service.

\subsection{Statistical analysis}

Each hypothesis will be tested on an intent-to-treat basis using mixed effects model repeated measures (MMRM) based estimates. These models incorporate all available data, including participants with missing data points, under the missing-at-random assumption without using biased techniques such as last observation carried forward [35]. Continuous outcomes will be tested with linear MMRM while categorical and binary outcomes will be analysed using binary mixed effects models. Patterns of missing data will be examined and tested for correlations with attrition, and then adjusted for in sensitivity analyses, also using MMRM.

The analysis plan has factored for contamination effects in the study design by ensuring participants allocated to the usual care group do not have direct contact with the PHNC. Nevertheless, this group will complete self-report items at 24 months assessing whether participants in both groups received cardiovascular monitoring, as an indicator for both 'dosage' effects (amount of intervention received) and whether any contamination may have occurred. This self-report data will be combined with service use data in moderator analyses to determine whether a dosage effect was evident. Additional potential confounders and/or moderators of outcome such as age, gender, stage of diagnosis (first episode psychosis vs established psychotic disorder), medications and pre-existing comorbidities will be tested and controlled for in secondary analyses using MMRM, testing three-way interactions between moderator, time and condition. We will report findings based on CONSORT guidelines.

\subsection{Power calculations}

Reviews of similar interventions have shown medium to large effects (range $f=0.2-0.8$ ) on change in these outcomes [36-38]. Hence to detect an effect size of $\mathrm{f}=0.2$ in primary and secondary outcome measures between groups at 24 months with nine repeated measures, 56 participants are required in each arm to achieve $80 \%$ power using an alpha level of 0.05 . This number has been inflated ( 80 participants per arm) to account for up to $30 \%$ attrition from assessments. Hence a target of $N=160$ total participants will be recruited into the RCT. Assuming a correlation among repeated measures of 0.5 , this study will be sufficiently powered to detect significant changes in all outcomes.

\section{Discussion}

\subsection{Summary}

This paper describes the protocol for the implementation and evaluation of a PHNC service to be delivered alongside usual care for consumers with psychosis. In particular, we will evaluate the PHNC service relative to usual care with respect to cardiometabolic health, consumer experience of care, and cost-effectiveness. This trial will be significant as the first large scale implementation of such a role in Australia, and the first to include consumer evaluation and cost-effectiveness analysis.

The prevalence and severity of physical health comorbidities in consumers with psychosis has been well documented. However there remains a critical implementation gap around how to deliver a high standard of evidence-based effective physical healthcare within the current Australian mental health service and policy landscape. This project is based on the best available evidence on physical health service delivery and uses a robust, innovative methodology to investigate the expected benefits in Burden of Disease risk factors, consumer experience of care, and cost-effectiveness to directly address this implementation gap.

The main outcome of this project will be a fully evaluated model of service that directly addresses the nationally-significant health services priority of sustainably improving consumer physical health outcomes. The model is aligned to local, national and international consensus on best-practice to maximise health and translational impact. Further, robust evaluation data will support the model's benefits on risk factors and disease prevalence, consumer experience of care and cost-effectiveness. Specifically, the model is expected to more closely align to best-practice service standards, delivering comprehensive assessment and more efficient and effective linkage to preventative care to improve the physical health of 690,000 consumers nationally [2]. If scaled up, this intervention has the potential to reduce comorbid burden of disease risk and prevalence by up to $60 \%$ [36], with proportionate reductions in the $\$ 15$ billion annual impact of comorbid physical illness in this population [2]. Such economic savings may occur largely due to longterm reductions in national healthcare system costs, which will of significant interest to policymakers. For service providers, the initial costs associated with implementing the PHNC service will be offset by substantial ongoing cost savings in provisioning health services in the medium-to-long term. These outcomes can be readily translated into clinical health services to transform mental health service delivery by giving equal importance to comorbid physical health through evidencebased specialist care. Critically, the PHNC intervention meets the consumer-identified preference of having both physical and mental health specialist care embedded within the mental health service.

Project outcomes also directly support the NHMRC Case for Action on Improving the Prevention and Management of Chronic Disease Among People with Mental Illness [39] and aligns with multiple National Health 


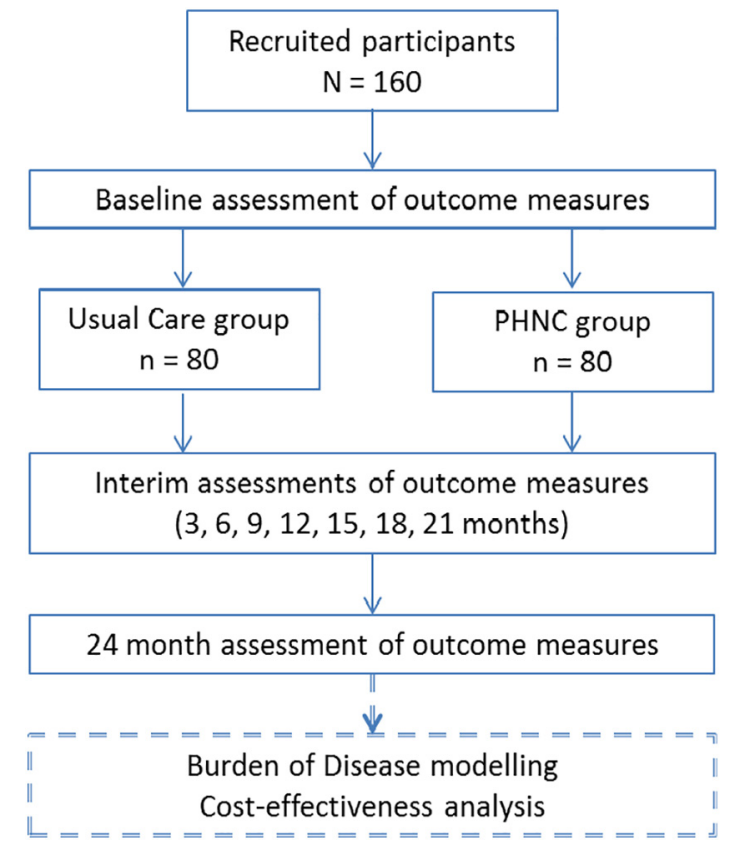

Fig. 1. Flow through of participant in the study.

Priority Areas including: Cardiovascular Health and Stroke; Diabetes Mellitus; Mental Health; and Obesity. These outcomes also align with policy and recommendations from the Fifth National Mental Health Plan [7], Royal Australian and New Zealand College of Psychiatrists [13], and the National Mental Health Commission [8]. They are expected to have a significant impact on health policy given that the National Mental Health Commission are currently seeking solutions for this priority issue [16] Fig. 1.

This project is innovative as it supports the NHMRC Statement on Consumer Participation in Health and Medical Research. Our project team includes personnel who are academically-trained consumer researchers with lived experience of mental health service use. The project methodology and outcomes are also highly innovative because they are among the first to consider the importance of consumer experience of care in mental health service delivery and its influence on disease risk, prevalence and cost-effectiveness [38,40].

\subsection{Translation to usual care}

A key outcome from this project will be the translation of the PHNC service to usual care. The implementation will involve case managers referring new consumers for an initial consultation with the PHNC. During the consultation, the PHNC will conduct a comprehensive assessment of cardiometabolic health and health behaviours. Cardiometabolic risks will be identified using the Positive Cardiometabolic Health treatment framework [23]. The PHNC and consumer will discuss any identified cardiometabolic risks based on pre-existing comorbidities, medication use and other risk factors, then identify risk management strategies using a shared decision-making approach [41-43]. Once management decisions are agreed upon, the PHNC will support the consumer in making an appointment, coordinating information and results with referred services, providing appointment reminders to the consumer, and following up on attendance and results. Progress against agreed goals will be monitored in shorter monthly telephone follow-up consultations, with cardiometabolic assessments being repeated quarterly. This differs from the study described in this paper in that the PHNC would complete all assessments, whereas in this trial the Clinical Research Assistant will perform baseline assessments. This separation of tasks is to ensure the methodological integrity of the trial to reduce contamination effects but would be unnecessary for translation into usual care.

\section{Competing interests}

The authors declare they have no competing interests.

\section{Author's contributions}

$\mathrm{BH}$ is the grant awardee and lead investigator on this project. BH led the conception and design of the study. JC contributed to the study design, specifically the implementation of the Positive Cardiometabolic Health Framework. MB, JB and AW contributed to study design, specifically service integration and consumer experience evaluation components. JG, LM and VP contributed to design of the outcome evaluation and the cost effectiveness aspect of the study including the method for using burden of disease data to estimate outcomes now and 20 years into the future. TN, PB and CPP guided the statistical design of this project. RS contributed to preliminary trial design. BS contributed to study design and study implementation. All authors have sufficiently contributed to the preparation of this manuscript to meet the authorship requirements. All authors read and approved the final version of this manuscript for submission.

\section{Acknowledgements}

This research is funded by the National Health and Medical Research Council (APP No: 1139596). PB is supported by NHMRC fellowship 1083311. The content is solely the responsibility of the authors and does not necessarily represent the official views of the funding agency. The funder has no role in the study design, data collection, analysis, or interpretation of the data, nor did they contribute to the preparation of this manuscript.

\section{References}

[1] Royal Australian and New Zealand College of Psychiatrists, Keeping Body and Mind Together. 2015, RANZCP: Melbourne.

[2] Royal Australian and New Zealand College of Psychiatrists, The economic cost of serious mental illness and comorbidities in Australia and New Zealand. 2016, RANZCP: Sydney.

[3] D. Lawrence, K.J. Hancock, S. Kisely, The gap in life expectancy from preventable physical illness in psychiatric patients in Western Australia: retrospective analysis of population based registers, Br. Med. J. 346 (2013).

[4] T.M. Laursen, Life expectancy among persons with schizophrenia or bipolar affective disorder, Schizophr. Res. 131 (1) (2011) 101-104.

[5] C.A. Galletly, et al., Cardiometabolic risk factors in people with psychotic disorders: the second Australian national survey of psychosis, Aust. N. Z. J. Psychiatry 46 (8) (2012) 753-761.

[6] J. Magarey, New look at mental illness: bill tops \$100bn, in The Australian, Sydney, The Australian, 2015.

[7] Department of Health, Fifth National Mental Health Plan, Department of Health: Canberra., 2017.

[8] National Mental Health Commission, Equally Well: Consensus Statement to improve the physical health and wellbeing of people living with mental illness in Australia. 2016, National Mental Health Commission: Sydney.

[9] Australian Capital Territory Health Directorate, ACT Mental Health Services Plan 2009-2014, (2017) (Canberra).

[10] Australian Capital Territory Health Directorate, ACT Chronic Conditions Strategy Improving Care and Support: 2013-2018 2013:Canberra.

[11] D. Shiers, T. Bradshaw, J. Campion, Health inequalities and psychosis: time for action, Br. J. Psychiatry 207 (6) (2015) 471-473.

[12] D. Shiers, J. Curtis, Cardiometabolic health in young people with psychosis, The Lancet Psychiatry 1 (7) (2014) 492-494.

[13] C. Galletly, et al., Royal Australian and New Zealand College of Psychiatrists clinical practice guidelines for the management of schizophrenia and related disorders, Aust N Z J Psychiatry 50 (5) (2016) 410-472.

[14] B. Organ, E. Nicholson, D. Castle, Implementing a physical health strategy in a mental health service, Australasian Psychiatry 18 (5) (2010) 456-459.

[15] B. McKenna, et al., The effectiveness of specialist roles in mental health metabolic monitoring: a retrospective cross-sectional comparison study, BMC Psychiatry 14 (1) (2014) 234.

[16] Australian National Mental Health Commission, Report of the National Review of Mental Health Programmes and Services: Contributing lives, thriving communities, (2014).

[17] M. De Hert, et al., Physical illness in patients with severe mental disorders. Ii. 
Barriers to care, monitoring and treatment guidelines, plus recommendations at the system and individual level, World Psychiatry 10 (2) (2011) 138-151.

[18] C. Blanner Kristiansen, et al., Promoting physical health in severe mental illness: patient and staff perspective, Acta Psychiatr. Scand. 132 (6) (2015) 470-478.

[19] B. Happell, et al., The cardiometabolic health nurse; physical health behaviour outcomes from a randomised controlled trial, Issues in Mental Health Nursing 35 (10) (2014) 768-775.

[20] S.N. Bleich, E. Özaltin, C.J. Murray, How does satisfaction with the health-care system relate to patient experience? Bull. World Health Organ. 87 (4) (2009) 271-278.

[21] P. Ryan, Integrated theory of health behavior change: background and intervention development, Clinical nurse specialist CNS 23 (3) (2009) 161.

[22] ICH, Statistical principles for clinical trials. 1998, International Council for Harmonisation of Technical Requirements for Pharmaceuticals for Human Use: Geneva, Switzerland.

[23] Curtis, J., K. Samaras, and H. Newall, Psychiatry Positive Cardio Metabolic Algorithm, H.E.T. Institute, Editor. 2012, NSW Government: Sydney.

[24] G. Deed, et al., General Practice Management of Type 2 Diabetes: 2014-15, (2014) (Melbourne).

[25] ABS, Australian Health Survey: Users' Guide, 2011-13, ABS, Editor. 2013, ABS Canberra.

[26] AIHW, Australian Burden of Disease Study: impact and causes of illness and death in Australia 2011, in Australian Burden of Disease Study series no. 3. 2016, AIHW: Canberra.

[27] S.T. Wong, J. Haggerty, Measuring patient experiences in primary health care: a review and classification of items and scales used in publicly-available questionnaires, Centre for Health Services and Policy Research (CHSPR) Publications (2013), https://open.library.ubc.ca/cIRcle/collections/ facultyresearchandpublications/52383/items/1.0048528.

[28] J. Richardson, et al., Modelling the utility of health states with the Assessment of Quality of Life (AQoL) $8 D$ instrument: overview and utility scoring algorithm. Research Paper 63, Center for Health Economics, 2011.

[29] Richardson, J., et al., Increasing the sensitivity of the AQoL inventory for evaluation of interventions affecting mental health, in Research paper. 2011, Centre for Health Economics: Melbourne.

[30] J. Richardson, et al., Validity and reliability of the Assessment of Quality of Life
(AQoL)-8D multi-attribute utility instrument, The Patient-Patient-Centered Outcomes Research 7 (1) (2014) 85-96.

[31] C. Mihalopoulos, et al., The economic analysis of prevention in mental health programs, Annu. Rev. Clin. Psychol. 7 (2011) 169-201.

[32] T. Vos, et al., Assessing Cost-Effectiveness in the Prevention of Non-Communicable Disease: Economic Evaluation Protocol, Brisbane, University of Queensland, 2007.

[33] Nichols, M., et al., Australian heart disease statistics. Overweight, obesity and cardiovascular disease - past, present and future. 2015, National Heart Foundation of Australia: Melbourne.

[34] D.O. Health, D.O. Health (Ed.), Guidelines for preparing a submission to the Pharmaceutical Benefits Advisory Committee, Commonwealth of Australia: Canberra, 2016.

[35] G. Verbeke, Linear mixed models for longitudinal data, in Linear mixed models in practice, Springer, 1997, pp. 63-153.

[36] J. Curtis, et al., Evaluating an Individualized Lifestyle and Life Skills Intervention to Prevent Antipsychotic-Induced Weight Gain in First-Episode Psychosis, Early Intervention in Psychiatry (2015).

[37] M.I. Fernandez-San-Martin, et al., The effectiveness of lifestyle interventions to reduce cardiovascular risk in patients with severe mental disorders: Meta-analysis of intervention studies, Community Ment. Health J. 50 (1) (2014) 81-95.

[38] S.R. de Bruin, et al., Comprehensive care programs for patients with multiple chronic conditions: a systematic literature review, Health Policy 107 (2) (2012) $108-145$.

[39] A. Wilson, et al., Case for Action proposal: Improving the prevention and management of chronic disease among people with mental illness, (2015) (Canberra).

[40] D.W. Bradford, et al., An evidence synthesis of care models to improve general medical outcomes for individuals with serious mental illness: a systematic review, J. Clin. Psychiatry 74 (8) (2013) e754-e764.

[41] B. Happell, C. Platania-Phung, D. Scott, Mental Health Nurse Incentive Program: Facilitating physical health care for people with mental illness? Int. J. Ment. Health Nurs. 22 (5) (2013) 399-408.

[42] B. Happell, D. Scott, C. Platania-Phung, Perceptions of barriers to physical health care for people with serious mental illness: a review of the international literature Issues in Mental Health Nursing 33 (11) (2012) 752-761.

[43] B.G. Druss, R.A. Rosenheck, Locus of mental health treatment in an integrated service system, Psychiatr. Serv. 51 (7) (2000) 890-892. 